Real Analysis Exchange

Vol. 30(2), 2004/2005, pp. 783-794

Ch. Rini Indrati, Department of Mathematics, Gadjah Mada University,

Sekip Utara, Yogyakarta, Indonesia.

email: chrini.indrati@lycos.com or rinii@ugm.ac.id

Lee Peng Yee, MME, National Institute of Education, 1 Nanyang Walk,

Singapore 637616. email: pylee@nie.edu.sg

\title{
DOMINATED CONVERGENCE THEOREM INVOLVING SMALL RIEMANN SUMS
}

\begin{abstract}
We define two interval functions $U_{\delta}$ and $V_{\delta}$ using Riemann sums of Henstock integrable functions, as major and minor functions. Then we formulate two dominated convergence theorems for the Henstock integral in the $n$-dimensional space.
\end{abstract}

\section{Introduction.}

The Henstock integral is well-known. Convergence theorems for the integral have been proved using conditions involving small Riemann sums. See, for example, $[1,2,4,6]$. In this paper, we define two interval functions $U_{\delta}$ and $V_{\delta}$ using Riemann sums of Henstock integrable functions as major and minor functions. Then we formulate two dominated convergence theorems for the Henstock integral in the $n$-dimensional space.

In this paper, we consider real-valued functions defined on a cell of $\mathbb{R}^{n}$. In what follows, $E$ stands for a cell (a non-degenerate closed interval) in $\mathbb{R}^{n}$, and is fixed. The volume of a cell $E$ will be represented by $|E|$. Here, $\mathbb{R}^{n}$ is a normed space with respect to the norm \|\|$_{\infty}$; i.e., for $\mathbf{x}=\left(x_{1}, x_{2}, \ldots, x_{n}\right) \in \mathbb{R}^{n}$,

$$
\|\mathbf{x}\|_{\infty}=\max \left\{\left|x_{k}\right|: 1 \leq k \leq n\right\} .
$$

If $E$ is a cell and $\delta$ is a positive function on $E$, for $\mathbf{x} \in E$, then

$$
B(\mathbf{x}, \delta(\mathbf{x}))=\left\{\mathbf{y}:\|\mathbf{x}-\mathbf{y}\|_{\infty}<\delta(\mathbf{x})\right\}
$$

Key Words: Henstock Integral, $\delta$-fine partition, non-absolute partition, Locally Small Riemann Sum, Functionally Small Riemann Sum, $U_{\delta}, V_{\delta}$, Uniformly Strong Lusin Condition, Equi-Henstock integrability.

Mathematical Reviews subject classification: Primary 26A39.

Received by the editors May 8, 2004

Communicated by: Peter Bullen 
is called an open ball with center at $\mathbf{x}$ and radius $\delta(\mathbf{x})$.

A collection of cells, $\left\{I_{i}: i=1,2, \ldots\right\}$, is called non-overlapping if $I_{i}^{o} \cap I_{j}^{o}$ $=\emptyset$ for $i \neq j$, where $I_{i}^{o}$ and $I_{j}^{o}$ denote the interiors of $I_{i}$ and $I_{j}$, respectively. Further, a collection of finite non-overlapping cells $\mathcal{D}=\{I\}$, with $\cup_{I \in \mathcal{D}} I=$ $E$, is called a partition of $E$. A collection

$$
\mathcal{D}=\{(I, \mathbf{x})\}=\left\{\left(I_{1}, \mathbf{x}_{1}\right),\left(I_{2}, \mathbf{x}_{2}\right), \ldots,\left(I_{p}, \mathbf{x}_{p}\right)\right\}
$$

is called $\delta$-fine partition of a cell $E$ if $E=\cup_{i=1}^{p} I_{i}, \mathbf{x}_{i} \in I_{i} \subseteq B\left(\mathbf{x}_{i}, \delta\left(\mathbf{x}_{i}\right)\right)$, and $I_{i}^{o} \cap I_{j}^{o}=\emptyset, i \neq j, i=1,2, \ldots, p$. Furthermore, $(I, \mathbf{x}) \in \mathcal{D}$ is called $\delta$-fine interval with associated point $\mathbf{x}$. If $\cup_{i=1}^{p} I_{i} \subseteq E$, then the partition is called $\delta$-fine partial partition of $E$.

A function $f$ defined on a cell $E$ is said to be Henstock integrable on a cell $E$, written $f \in H(E)$, if there is a number A such that for any $\epsilon>0$ there is a positive function $\delta$ on $E$ such that for any $\delta$-fine partition $\mathcal{D}=\{(I, \mathbf{x})\}=$ $\left\{\left(I_{1}, \mathbf{x}_{1}\right),\left(I_{2}, \mathbf{x}_{2}\right), \ldots,\left(I_{p}, \mathbf{x}_{p}\right)\right\}$ of $\mathrm{E}$, we have

$$
\left|(\mathcal{D}) \sum f(\mathbf{x})\right| I|-A|<\epsilon \text {. }
$$

Here $(\mathcal{D}) \sum f(\mathbf{x})|I|=\sum_{i=1}^{p} f\left(\mathbf{x}_{i}\right)\left|I_{i}\right|$. If a function $f$ is Henstock integrable on a cell $E$, then the integral value of $f$ on $E$ is unique. Furthermore, the number $A$ is called the integral value of $f$ on $E$ and will be written by

$$
A=(H) \int_{E} f
$$

If we only want to know whether a function $f$ is Henstock integrable on a cell $E$ without using its integral value, we may use Cauchy's Criterion. More precisely, a function $f$ is Henstock integrable on a cell $E$ if and only if for any $\epsilon>0$ there is a positive function $\delta$ on $E$ such that for any two $\delta$-fine partitions $\mathcal{D}_{1}$ and $\mathcal{D}_{2}$ of $E$, we have

$$
\left|\left(\mathcal{D}_{1}\right) \sum f(\mathbf{x})\right| I\left|-\left(\mathcal{D}_{2}\right) \sum f(\mathbf{x})\right| I||<\epsilon .
$$

Standard properties of the Henstock integral in the $n$-dimensional space can be found in $[2,3]$.

If $f$ is Henstock integrable on $E$ and $I$ is a subcell of $E$, then $f$ is Henstock integrable on $I$. Let $F(I)$ denote the integral of $f$ on $I \subseteq E$. Then $F$ is called the primitive of $f$ on $E$ and Henstock's Lemma holds. More precisely, a function $f$ defined on $E$ is Henstock integrable with primitive $F$ if and only if for every $\epsilon>0$ there is a positive function $\delta$ on $E$ such that for any $\delta$-fine partial partition $\mathcal{D}$ of $E$, we have

$$
\left|(\mathcal{D}) \sum(f(\mathbf{x})|I|-F(I))\right|<\epsilon .
$$




\section{A Dominated Convergence Theorem.}

A measurable function $f$ defined on $E$ has Locally Small Riemann Sums, or the LSRS property, if for every $\epsilon>0$ there is a positive function $\delta$ such that for any $\mathbf{t} \in E$ we have

$$
\left|(\mathcal{D}) \sum f(\mathbf{x})\right| I||<\epsilon
$$

for every $\delta$-fine partition $\mathcal{D}=\{(I, \mathbf{x})\}$ of a cell $C \subseteq B(\mathbf{t}, \delta(\mathbf{t}))$ and $\mathbf{t} \in C$.

The following two theorems are known [2].

Theorem 2.1. If $f$ has the LSRS property on $E$, then there is a positive function $\delta$ on $E$ such that $\left\{(\mathcal{D}) \sum f(\mathbf{x})|I|: \mathcal{D}\right.$ is a $\delta$-fine partition of $\left.E\right\}$ is bounded.

We define the following interval functions, if they exist,

$$
U_{\delta}(I)=\sup \left\{f_{k}(\mathbf{x})|I|: k=1,2, \ldots,(I, \mathbf{x}) \text { is } \delta-\text { fine }\right\}
$$

and

$$
V_{\delta}(I)=\inf \left\{f_{k}(\mathbf{x})|I|: k=1,2, \ldots,(I, \mathbf{x}) \text { is } \delta-\text { fine }\right\} .
$$

Given a sequence $\left\{f_{k}\right\}$, we define the sequence of measurable functions on a cell $E$ has uniformly locally small Riemann sums, or the ULSRS property, if the conditions for LSRS hold with $f$ replaced by $f_{k}$ and $\delta$ independent of $k$. We remark that if $\left\{f_{k}\right\}$ has the ULSRS property, then in views of Theorem 2.1 , there exists a positive function $\delta$ such that both $U_{\delta}$ and $V_{\delta}$ exist for $I \subseteq E$.

The functions $U_{\delta}$ and $V_{\delta}$ serve as major and minor functions for $f_{k}, k=$ $1,2,3, \ldots$. Note that here $f_{k}, k=1,2, \ldots$, are point functions, whereas $U_{\delta}$ and $V_{\delta}$ are interval functions depending on $f_{k}$. We use them to formulate a convergence theorem below.

Theorem 2.2. If the following conditions are satisfied:

(i) $\left\{f_{k}\right\}$ is a sequence of Henstock integrable functions on $E$ with $f_{k}(\mathbf{x}) \rightarrow$ $f(\mathbf{x})$ almost everywhere in $E$,

(ii) for any $\epsilon>0$ there is an $\eta>0$ such that for every open set $G$ with $|G|<$ $\eta$ there is a positive function $\delta$ such that for every partition $\mathcal{D}=\{I\}$ with $I \subseteq G, U_{\delta}(I)$ and $V_{\delta}(I)$ exist and we have

$$
(\mathcal{D}) \sum\left\{U_{\delta}(I)-V_{\delta}(I)\right\}<\epsilon,
$$


then $f$ is Henstock integrable on $E$ and

$$
\lim _{k \rightarrow \infty}(H) \int_{E} f_{k}=(H) \int_{E} f
$$

Proof. We may assume that $f_{k}(\mathbf{x})$ converges to $f(\mathbf{x})$ everywhere in $E$. Let $\epsilon>0$ be given, and $A_{k}=\int_{E} f_{k}$ for every $k$. For every $k$, since $f_{k}$ is Henstock integrable on $E$, there exists a positive function $\delta_{k}$ on $E$ such that for any $\delta_{k}$-fine partition $\mathcal{D}$ of $E$

$$
\left|(\mathcal{D}) \sum f_{k}(\mathbf{x})\right| I\left|-A_{k}\right|<\epsilon
$$

For every $\delta$-fine interval $(I, \mathbf{x})$, we have

$$
V_{\delta}(I) \leq f_{k}(\mathbf{x})|I| \leq U_{\delta}(I)
$$

for every $k$. Further, (4) implies that for every $\delta$-fine interval $(I, \mathbf{x})$,

$$
V_{\delta}(I) \leq f(\mathbf{x})|I| \leq U_{\delta}(I)
$$

For every $\eta_{*}>0$, there is an open set $G_{*} \subset E$ with $\left|G_{*}\right|<\eta_{*}$ such that there exists a positive integer $k_{o}$ such that for every $k, m \geq k_{o}$ and for every $\mathbf{x} \in E \backslash G_{*}$, we have

$$
\left|f_{k}(\mathbf{x})-f_{m}(\mathbf{x})\right| \leq \frac{\epsilon}{|E|}
$$

By condition (ii) in Theorem 2.2, there is an $\eta>0$ such that for every $G$ with $|G|<\eta$ there is a positive function $\delta$ such that for every partition $\mathcal{D}=\{I\}$ with $I \subseteq G$ and for all $k$ and $m$, we have

$$
\left|(\mathcal{D}) \sum_{\mathbf{x} \in G} f_{k}(\mathbf{x})\right| I\left|-(\mathcal{D}) \sum_{\mathbf{x} \in G} f_{m}(\mathbf{x})\right| I|| \leq(\mathcal{D}) \sum\left\{U_{\delta}(I)-V_{\delta}(I)\right\}<\epsilon .
$$

Take $\eta^{*}=\eta$, then there is a set $G^{*} \subset E$ with $\left|G^{*}\right|<\eta^{*}$ such that (6) and (7) are satisfied. For $m, k \geq k_{o}$, take $\delta_{*}(\mathbf{x})=\min \left\{\delta(\mathbf{x}), \delta_{k}(\mathbf{x}), \delta_{m}(\mathbf{x})\right\}$. Modify the positive function $\delta_{*}$ such that for every $\mathbf{x} \in G$, we have $B\left(\mathbf{x}, \delta_{*}(\mathbf{x})\right) \subseteq G$. Therefore, for every $k, m \geq k_{o}$ and for every $\delta_{*}$-fine partition $\mathcal{D}$ of $E$, it follows from (3), (4), (6), and (7), we get 


$$
\begin{aligned}
\left|A_{k}-A_{m}\right| \leq & \left|A_{k}-(\mathcal{D}) \sum f_{k}(\mathbf{x})\right| I \mid \\
& +\left|(\mathcal{D}) \sum_{\mathbf{x} \notin G^{*}} f_{k}(\mathbf{x})\right| I\left|-(\mathcal{D}) \sum_{\mathbf{x} \notin G^{*}} f_{m}(\mathbf{x})\right| I \mid \\
& +\left|(\mathcal{D}) \sum_{\mathbf{x} \in G^{*}} f_{k}(\mathbf{x})\right| I\left|-(\mathcal{D}) \sum_{\mathbf{x} \in G^{*}} f_{m}(\mathbf{x})\right| I \mid \\
& +\left|(\mathcal{D}) \sum f_{m}(\mathbf{x})\right| I\left|-A_{m}\right|<4 \epsilon .
\end{aligned}
$$

So, the sequence $\left\{A_{k}\right\}$ is a Cauchy sequence. $A=\lim _{k \rightarrow \infty} A_{k}$ exists. It remains to prove that $f$ is Henstock integrable on $E$ and $A=(H) \int_{E} f$. Since $A=\lim _{k \rightarrow \infty} A_{k}$, there exists a positive number $k_{*}$ such that for $k \geq k_{*}$,

$$
\left|A_{k}-A\right|<\epsilon
$$

Put $K=\max \left\{k_{o}, k_{*}\right\}$. Then for $k \geq K$ and following the same argument above with $m \rightarrow \infty$, we obtain

$$
\left|A-(\mathcal{D}) \sum f(\mathbf{x})\right| I||<4 \epsilon .
$$

Example. Let $h, g, f_{k}, k=1,2,3, \ldots$, be Henstock integrable functions on $[a, b]$ with $f_{k}(\mathbf{x}) \rightarrow f(\mathbf{x})$ almost everywhere in $[a, b]$. If for every $k, h(\mathbf{x}) \leq$ $f_{k}(\mathbf{x}) \leq g(\mathbf{x})$ almost everywhere in $[a, b]$, then condition (ii) in Theorem 2.2 is satisfied.

Next, we shall prove a connection of Theorem 2.2 with FSRS in Theorem 2.6 and Theorem 3.4. The proof of Theorem 2.6 needs some concepts below. A sequence of functions $\left\{F_{k}\right\}$ is said to satisfy Uniformly Strong Lusin, or the USL condition, if for every $\epsilon>0$ and every set $S$ of measure zero there exists a positive function $\delta$ on $E$, independent of $k$, such that for any $\delta$-fine partial partition $\mathcal{D}=\{(I, \mathbf{x})\}$, with $\mathbf{x} \in S$, and for all $k$

$$
(\mathcal{D}) \sum\left|F_{k}(I)\right|<\epsilon \text {. }
$$

If $F_{k}=F$ for all $k$, then $F$ is said to satisfy the strong Lusin condition. A sequence $\left\{f_{k}\right\}$ is said to be equi-Henstock integrable on a cell $E$ if for every $\epsilon>0$ there is a positive function $\delta$ on $E$, independent of $k$, such that for any $\delta$-fine partition $\mathcal{D}=\{(I, \mathbf{x})\}$ of $E$ and for every $k$,

$$
\left|(\mathcal{D}) \sum f_{k}(\mathbf{x})\right| I\left|-A_{k}\right|<\epsilon
$$

The proof of Lemma 2.4 needs Lemma 2.3. 
Lemma 2.3. [6] Let $f_{k}, k=1,2,3, \ldots$, be Henstock integrable on a cell $E$ with the primitives $F_{k}, k=1,2,3, \ldots$, respectively. If there is a non-negative Lebesgue integrable function $g$ on $E$ such that $\left|f_{k}(\mathbf{x})\right| \leq g(\mathbf{x})$ almost everywhere for every $k$, and $f_{k}(\mathbf{x}) \rightarrow f(\mathbf{x})$ almost everywhere in $E$, then $\left\{F_{k}\right\}$ satisfies the USL condition on $E$ and $\left\{f_{k}\right\}$ is equi-Henstock integrable on $E$.

Lemma 2.4. Let $f_{k}, k=1,2,3, \ldots$, be Henstock integrable on a cell $E$ and let $g$ be a non-negative Lebesgue integrable function on a cell $E$ such that $\left|f_{k}(\mathbf{x})\right| \leq$ $g(\mathbf{x})$ almost everywhere for every $k$, and $f_{k}(\mathbf{x}) \rightarrow f(\mathbf{x})$ almost everywhere in $E$, then for every $\epsilon>0$ there exist a positive function $\delta$ on $E$ and a positive integer $k_{1}$ such that for every $\delta$-fine partition $\mathcal{D}$ of $E$ and for every $k \geq k_{1}$

$$
\left|(\mathcal{D}) \sum_{\left|f_{k}(\mathbf{x})\right| \leq g(\mathbf{x})} f_{k}(\mathbf{x})\right| I\left|-(\mathcal{D}) \sum_{|f(\mathbf{x})| \leq g(\mathbf{x})} f(\mathbf{x})\right| I||<\epsilon .
$$

Proof. The proof is known. We sketch the proof as follows. Put

$$
\left.f_{k}^{*} \mathbf{x}\right)= \begin{cases}f_{k}(\mathbf{x}) & \left|f_{k}(\mathbf{x})\right| \leq g(\mathbf{x}) \\ 0 & \text { otherwise }\end{cases}
$$

for $k=1,2,3, \ldots$

$$
f^{*}(\mathbf{x})= \begin{cases}f(\mathbf{x}) & |f(\mathbf{x})| \leq g(\mathbf{x}) \\ 0 & \text { otherwise }\end{cases}
$$

then using the Dominated Convergence Theorem we obtain that

$$
f^{*} \text { is Henstock integrable and }(H) \int_{E} f^{*}=\lim _{k \rightarrow \infty}(H) \int_{E} f_{k}^{*} \text {. }
$$

The rest of proof can be done by Lemma 2.3.

A measurable function $f$ defined on $E$ has Functionally Small Riemann Sums, or the FSRS property, if for every $\epsilon>0$ there exist a positive function $\delta$ and a non-negative Lebesgue integrable $g$ on $E$ such that for any $\delta$-fine $\mathcal{D}$ of $E$, we have

$$
\left|(\mathcal{D}) \sum_{|f(\mathbf{x})|>g(\mathbf{x})} f(\mathbf{x})\right| I||<\epsilon .
$$

Theorem 2.5. [5] If $f$ is Henstock integrable on E, then $f$ has the FSRS property on $E$.

A sequence $\left\{f_{k}\right\}$ of measurable functions has uniformly functionally small Riemann sums, or the UFSRS property, if the conditions for FSRS hold with $f$ replaced by $f_{k}$ and both $g$ and $\delta$ independent of $k$. 
Theorem 2.6. If the conditions in Theorem 2.2 hold, then $\left\{f_{k}\right\}$ has the UFSRS property.

Proof. Let $\epsilon>0$ be given. Since $f$ is Henstock integrable on $E$, then $f$ has the FSRS property. So, there exists a positive function $\delta_{*}$ on $E$ and a nonnegative Lebesgue integrable function $g$ such that for every $\delta_{*}$-fine partition $\mathcal{D}$ of $E$,

$$
\left|(\mathcal{D}) \sum_{|f(\mathbf{x})|>g(\mathbf{x})} f(\mathbf{x})\right| I||<\epsilon .
$$

By condition (ii) in Theorem 2.2, there is an $\eta>0$ such that for every $G$ with $|G|<\eta$ there is a positive function $\delta$ such that for every partition $\mathcal{D}=\{I\}$ with $I \subseteq G$, we have

$$
\left|(\mathcal{D}) \sum_{\mathbf{x} \in G} f_{k}(\mathbf{x})\right| I\left|-(\mathcal{D}) \sum_{\mathbf{x} \in G} f(\mathbf{x})\right| I|| \leq(\mathcal{D}) \sum\left\{U_{\delta}(I)-V_{\delta}(I)\right\}<\epsilon .
$$

For every $\eta_{*}>0$, there is an open set $G_{*} \subset E$ with $\left|G_{*}\right|<\eta_{*}$ such that there exists a positive integer $k_{o}$ such that for every $k \geq k_{o}$ and for every $\mathrm{x} \in E \backslash G_{*}$, we have

$$
\left|f_{k}(\mathbf{x})-f(\mathbf{x})\right| \leq \frac{\epsilon}{|E|}
$$

From Lemma 2.4, there exists a positive function $\delta^{*}$ and a positive integer $k_{1}$ such that for every $\delta^{*}$-fine partition $\mathcal{D}$ of $E$ and for every $k \geq k_{1}$,

$$
\left|(\mathcal{D}) \sum_{\left|f_{k}(\mathbf{x})\right| \leq g(\mathbf{x})} f_{k}(\mathbf{x})\right| I\left|-(\mathcal{D}) \sum_{|f(\mathbf{x})| \leq g(\mathbf{x})} f(\mathbf{x})\right| I||<\epsilon .
$$

Take $K=\max \left\{k_{o}, k_{1}\right\}, \delta^{* *}(\mathbf{x})=\min \left\{\delta^{*}(\mathbf{x}), \delta_{*}(\mathbf{x}), \delta(\mathbf{x})\right\}$, and $\eta^{*}=\eta$. Then there exists $G^{*}$ with $\left|G^{*}\right|<\eta^{*}$ and (10) and (11) are satisfied. Then modify $\delta^{* *}$ such that for every $\mathbf{x} \in G^{*}, B\left(\mathbf{x}, \delta^{* *}(\mathbf{x})\right) \subseteq G^{*}$. Then, for any $\delta^{* *}$-fine partition $\mathcal{D}$ of $E$ and $k \geq K$, it follows from (10), (11), (12), and (9), we have

$$
\begin{aligned}
\left|(\mathcal{D}) \sum_{\left|f_{k}(\mathbf{x})\right|>g(\mathbf{x})} f_{k}(\mathbf{x})\right| I|| \leq & \left|(\mathcal{D}) \sum_{\mathbf{x} \in G^{*}} f_{k}(\mathbf{x})\right| I\left|-(\mathcal{D}) \sum_{\mathbf{x} \in G^{*}} f(\mathbf{x})\right| I|| \\
& +\left|(\mathcal{D}) \sum_{\mathbf{x} \notin G^{*}} f_{k}(\mathbf{x})\right| I\left|-(\mathcal{D}) \sum_{\mathbf{x} \notin G^{*}} f(\mathbf{x})\right| I|| \\
& +\left|(\mathcal{D}) \sum_{\left|f_{k}(\mathbf{x})\right| \leq g(\mathbf{x})} f_{k}(\mathbf{x})\right| I\left|-(\mathcal{D}) \sum_{|f(\mathbf{x})| \leq g(\mathbf{x})} f(\mathbf{x})\right| I|| \\
& +\left|(\mathcal{D}) \sum_{|f(\mathbf{x})|>g(\mathbf{x})} f(\mathbf{x})\right| I||<4 \epsilon .
\end{aligned}
$$


Modify $\delta^{* *}$ and $g$, if necessary, so that the above inequality holds for every $k$. Hence, $\left\{f_{k}\right\}$ has the UFSRS property.

We fail to prove the converse of Theorem 2.6. In order to establish the converse relation with UFSRS, we extend Theorem 2.2 further in the next section.

\section{Another Dominated Convergence Theorem.}

Let $E$ be a cell in an $n$-dimensional space. A partial partition $\mathcal{D}=\{(I, \mathbf{x})\}$ of $E$ is said to be non-absolute in an open set $G$ if there exists $\delta(\mathbf{x})>0$ for $\mathbf{x} \in E$ such that $\bigcup_{(I, \mathbf{x}) \in \mathcal{D}} I$ is the complement of a $\delta$-fine cover of $E \backslash G$. A $\delta$-fine cover of $E \backslash G$ is the union of the intervals

$$
I_{1}, I_{2}, I_{3}, \ldots, I_{p}
$$

such that $\left(I_{i}, \mathbf{x}_{i}\right)$ is $\delta$-fine with $\mathbf{x}_{i} \in E \backslash G$ for $i=1,2,3, \ldots, p$, and the union contains $E \backslash G$.

When $E=[a, b] \subset \mathbb{R}$, that a partial partition $\mathcal{D}$ is non-absolute in $G$ means: the union of the intervals from $\mathcal{D}$ in each component interval of $G$ is again an interval and not the union of disjoint components.

Theorem 3.1. If the following conditions are satisfied:

(i) $\left\{f_{k}\right\}$ is a sequence of Henstock integrable functions on $E$,

(ii) there exists an open set $G$ such that $f_{k}(\mathbf{x}) \rightarrow f(\mathbf{x})$ uniformly on $E \backslash G$ and for every $\epsilon>0$ there is a positive function $\delta$ such that for every non-absolute partition $\mathcal{D}=\{(I, \mathbf{x})\}$ in $G$ using $\delta$, we have

$$
\text { (D) } \sum\left\{U_{\delta}(I)-V_{\delta}(I)\right\}<\epsilon,
$$

then $f$ is Henstock integrable on $E$ and

$$
\lim _{k \rightarrow \infty}(H) \int_{E} f_{k}=(H) \int_{E} f .
$$

Proof. We may assume that $f_{k}(\mathbf{x})$ converges to $f(\mathbf{x})$ everywhere in $E$. Let $\epsilon>0$ be given, and $A_{k}=\int_{E} f_{k}$ for every $k$. For every $k$, since $f_{k}$ is Henstock integrable on $E$, there exists a function $\delta_{k}$ on $E$ such that for any $\delta_{k}$-fine partition $\mathcal{D}$ of $E$,

$$
\left|(\mathcal{D}) \sum f_{k}(\mathbf{x})\right| I\left|-A_{k}\right|<\epsilon
$$


For every $\delta$-fine interval $(I, \mathbf{x})$, we have

$$
V_{\delta}(I) \leq f_{k}(\mathbf{x})|I| \leq U_{\delta}(I)
$$

for every $k$. Further, (4) implies that for every $\delta$-fine interval $(I, \mathbf{x})$,

$$
V_{\delta}(I) \leq f(\mathbf{x})|I| \leq U_{\delta}(I)
$$

By condition (ii) in Theorem 3.1, there is a positive integer $k_{o}$ such that for every $\mathbf{x} \in E \backslash G$ and for every $k, m \geq k_{o}$,

$$
\left|f_{k}(\mathbf{x})-f_{m}(\mathbf{x})\right|<\frac{\epsilon}{|E|}
$$

Again from (ii) in Theorem 3.1, for every non-absolute partition $\mathcal{D}=\{(I, \mathbf{x})\}$ in $G$ using $\delta$, we have

$$
\left|(\mathcal{D}) \sum_{\mathbf{x} \in G} f_{k}(\mathbf{x})\right| I\left|-(\mathcal{D}) \sum_{\mathbf{x} \in G} f(\mathbf{x})\right| I|| \leq(\mathcal{D}) \sum\left\{U_{\delta}(I)-V_{\delta}(I)\right\}<\epsilon .
$$

For $m, k \geq k_{o}$, take $\delta_{*}(\mathbf{x})=\min \left\{\delta(\mathbf{x}), \delta_{k}(\mathbf{x}), \delta_{m}(\mathbf{x})\right\}$. Modify the positive function $\delta_{*}$ such that for every $\mathbf{x} \in G$, we have $B\left(\mathbf{x}, \delta_{*}(\mathbf{x})\right) \subseteq G$. Therefore, for every $k, m \geq k_{o}$ and for every $\delta_{*}$-fine partition $\mathcal{D}$ of $E$, by (13), (16), and (17), we obtain

$$
\begin{aligned}
\left|A_{k}-A_{m}\right| \leq & \left|A_{k}-(\mathcal{D}) \sum f_{k}(\mathbf{x})\right| I|| \\
& +\left|(\mathcal{D}) \sum_{\mathbf{x} \notin G} f_{k}(\mathbf{x})\right| I\left|-(\mathcal{D}) \sum_{\mathbf{x} \notin G} f_{m}(\mathbf{x})\right| I|| \\
& +\left|(\mathcal{D}) \sum_{\mathbf{x} \in G} f_{k}(\mathbf{x})\right| I\left|-(\mathcal{D}) \sum_{\mathbf{x} \in G} f_{m}(\mathbf{x})\right| I|| \\
& +\left|(\mathcal{D}) \sum_{m}(\mathbf{x})\right| I\left|-A_{m}\right|<4 \epsilon .
\end{aligned}
$$

So, the sequence $\left\{A_{k}\right\}$ is a Cauchy sequence. The rest of the proof follows in similar way as the proof of Theorem 2.2.

It is easy to see that the conditions in Theorem 2.2 imply those in Theorem 3.1 , but not conversely as shown in the example below.

Example. Let

$$
f(\mathbf{x})= \begin{cases}(-1)^{k+1} k & x \in\left(\frac{1}{k+1}, \frac{1}{k}\right], k=1,2,3, \ldots \\ 0 & x=0\end{cases}
$$




$$
f_{k}(\mathbf{x})= \begin{cases}f(\mathbf{x}) & x \in\left(\frac{1}{2 k+1}, 1\right] \\ 0 & \text { otherwise }\end{cases}
$$

for $k=1,2,3, \ldots$.

Take $G=[0, \eta)$ such that $|F(x)|<\epsilon$ for $0<x<\eta$. Then condition (ii) in Theorem 3.1 is satisfied using the above $G$. Note that condition (ii) in Theorem 2.2 is not satisfied.

The proof of Theorem 3.4 needs the definition and a property of uniformly absolutely continuous.

A family of functions $\left\{F_{k}\right\}, k=1,2, \ldots$, is said to be uniformly absolutely continuous on a cell $E \subset \mathbb{R}^{n}$ if for every positive $\epsilon$ there is a positive $\eta$ such that if $\mathcal{D}$ is partial partition of $E$ with $(\mathcal{D}) \sum|I|<\eta$, then

$$
(\mathcal{D}) \sum|F(I)|<\epsilon
$$

Lemma 3.2. [3] Let $f_{k}, k=1,2,3, \ldots$, be Henstock integrable on a cell $E$ with the primitives $F_{k}, k=1,2,3, \ldots$, respectively. If there is a non-negative Lebesgue integrable function $g$ on $E$ such that $\left|f_{k}(\mathbf{x})\right| \leq g(\mathbf{x})$ almost everywhere for every $k$, then $\left\{F_{k}\right\}$ is uniformly absolutely continuous on $E$.

Lemma 3.3. If $\left\{f_{k}\right\}$ has the UFSRS property on $E$, then $\left\{f_{k}\right\}$ is equiHenstock integrable on E.

Proof. The proof is standard and therefore omitted. See [6].

Theorem 3.4. If $\left\{f_{k}\right\}$ has the UFSRS property on $E$ and $f_{k}(\mathbf{x}) \rightarrow f(\mathbf{x})$ almost everywhere in E, then the condition (ii) in Theorem 3.1 holds.

Proof. Let $\epsilon>0$ be given. There exist a nonnegative Lebesgue integrable function $g$ and a positive function $\delta_{*}$ on $E$ such that for every $\delta_{*}$-fine partition $\mathcal{D}$ of $E$ and for every $k$,

$$
\left|(\mathcal{D}) \sum_{\left|f_{k}(\mathbf{x})\right|>g(\mathbf{x})} f_{k}(\mathbf{x})\right| I||<\epsilon .
$$

For every $k$, we define

$$
h_{k}(x)= \begin{cases}f_{k}(x) & \left|f_{k}(\mathbf{x})\right| \leq g(\mathbf{x}) \\ 0 & \text { otherwise }\end{cases}
$$

and then $\left\{h_{k}\right\}$ is a sequence of equi-Henstock integrable functions. Let $H_{k}$ be the primitive of $h_{k}$, for every $k$. So, there is a positive function $\delta^{*}$ on $E$ such 
that for every $\delta^{*}$-fine partial partition $\mathcal{D}$ of $E$ and for every $k$, by Henstock's Lemma,

$$
\left|(\mathcal{D}) \sum h_{k}(\mathbf{x})\right| I\left|-H_{k}(I)\right|<\epsilon
$$

Since $\left\{h_{k}\right\}$ is dominated by $g$, by Lemma $3.2,\left\{H_{k}\right\}$ is uniformly absolutely continuous on $E$. There is an $\eta>0$ such that for every partial partition $\mathcal{D}=\{I\}$ with $(\mathcal{D}) \sum|I|<\eta$, we have

$$
\text { (D) } \sum\left|H_{k}(I)\right|<\epsilon
$$

for every $k$. Since $f_{k}(\mathbf{x}) \rightarrow f(\mathbf{x})$ almost everywhere in $E$, then for $\eta>0$ above there is an open set $G$, with $|G|<\eta$, such that $f_{k}(\mathbf{x}) \rightarrow f(\mathbf{x})$ uniformly on $E \backslash G$. Put $\delta(\mathbf{x})=\min \left\{\delta_{*}(\mathbf{x}), \delta^{*}(\mathbf{x})\right\}$. Modify $\delta$ such that for every $\mathbf{x} \in G$, we have $B(\mathbf{x}, \delta(\mathbf{x})) \subseteq G$. Therefore, for every non-absolute partition $\mathcal{D}=\{(I, \mathbf{x})\}$ of $G$ using $\delta$, it follows from (19), (18), and (20), we have

$$
\begin{aligned}
(\mathcal{D}) & \left(f_{j}(\mathbf{x})|I|-f_{k}(\mathbf{x})|I|\right) \\
\leq & \left|(\mathcal{D}) \sum_{\left|f_{j}(\mathbf{x})\right| \leq g(\mathbf{x})} f_{j}(\mathbf{x})\right| I\left|-(\mathcal{D}) \sum_{\left|f_{k}(\mathbf{x})\right| \leq g(\mathbf{x})} f_{k}(\mathbf{x})\right| I|| \\
& +\left|(\mathcal{D}) \sum_{\left|f_{j}(\mathbf{x})\right|>g(\mathbf{x})} f_{j}(\mathbf{x})\right| I||+\left|(\mathcal{D}) \sum_{\left|f_{k}(\mathbf{x})\right|>g(\mathbf{x})} f_{k}(\mathbf{x})\right| I|| \\
\leq & \left.\left.\left|(\mathcal{D}) \sum h_{j}(\mathbf{x})\right| I \mid\right)-H_{j}(I)\right)|+|(\mathcal{D}) \sum H_{j}(I)|+|(\mathcal{D}) \sum H_{k}(I) \mid \\
& \left.+\mid(\mathcal{D}) \sum\left(H_{k}(I)-h_{k}(\mathbf{x})|I|\right)\right) \mid+2 \epsilon<6 \epsilon .
\end{aligned}
$$

Since the above inequality holds for all $j, k$, hence for any non-absolute partition $\mathcal{D}=\{(I, \mathbf{x})\}$ of $G$ using $\delta,(\mathcal{D}) \sum\left\{U_{\delta}(I)-V_{\delta}(I)\right\}$ is small. Therefore, condition (ii) in Theorem 3.1 holds.

In conclusion, if the conditions in Theorem 2.2 hold, then the sequence $\left\{f_{k}\right\}$ has the UFSRS property. Conversely, if $\left\{f_{k}\right\}$ has the UFSRS property, then a weaker condition follows, namely condition (ii) of Theorem 3.1.

Acknowledgments. The authors are indebted to Professor P. S. Bullen for his help during the preparation of this paper, and to the referee for valuable suggestions. 


\section{References}

[1] R. Henstock, Lectures on the theory of integration, World Scientific, (1988).

[2] Lee P. Y., Lanzhou lectures on Henstock integration, World Scientific, (1989).

[3] Lee P. Y. and R. Výborný, Integral: an easy approach after Kurzweil and Henstock, Cambridge University Press, (2000).

[4] Y. Lin, On the generalized convergence theorems for Thomson's $\mathcal{B}$-integral in $\mathbb{R}^{m}$, Real Anal. Exchange, 21(1) (1995/96), 365-379.

[5] Ch. R. Indrati, The consequence of controlled densed theorem of HenstockKurzweil integral in n-dimensional Euclidean space, Proceeding of the Third Asian Mathematical Conference / AMC2000 held 23 - 27 October 2000 in Manila, The Philippines, (2002).

[6] Ch. R. Indrati, Convergence theorems for the Henstock integral involving small Riemann sums, Real Anal. Exchange, 29(1) (2003/04), 481-488. 